\title{
New UV Curable Acrylated Urethane-Oligoesters Derived From Poly(Ethylene Terephthalate) PET Waste
}

Medhat S. Farahat Khedr ( $\sim$ mfarahat2007@gmail.com )

University of North Florida

\section{Research Article}

Keywords: Polymer recycling, value-added recycled products, glycolysis of PET waste, UV curable monomers, urethane ester acrylated oligomers

Posted Date: June 30th, 2021

DOI: https://doi.org/10.21203/rs.3.rs-668530/v1

License: (a) (i) This work is licensed under a Creative Commons Attribution 4.0 International License.

Read Full License 


\section{Abstract}

The glycolysis products of polyethylene terephthalate (PET) waste represent a potential source for many value-added products that contain terephthalate repeating units in their backbones. Terephthalate repeating units were not attained directly from terephthalic acid due to its high melting point in addition to its tendency to sublime before it reacts. Glycolysis of PET provides an excellent solution for recycling polymer waste and constitutes a substantial starting material for manufacturing materials with high mechanical stress, such as unsaturated polyesters and polyurethane products. In this study, PET was first depolymerized by glycolysis, and glycolyzed products were then dimerized by reaction with toluene diisocyanate TDI with half equivalence of their hydroxyl groups for the purpose of inserting urethane blocks into the oligomer structure. The remaining half equivalence of terminal hydroxyl groups was modified into acrylate groups by an acrylation reaction. The acrylated oligo urethane ester products were crosslinked with different co-monomers and tested for UV curability and mechanical properties, and they showed outstanding results.

\section{Introduction}

Polyethylene terephthalate (PET) has been a well-known packaging material for many decades and is considered the world's top choice for many foods and beverages because of its hygienicity, high strength, light weight and shatterproofness. The highest application of PET lies in packaging carbonated soft drinks and water as a chemically safe material. The production of synthetic fibers from PET polymers fulfills more than half of the world's demand. PET was first synthesized in the United States by the mid1940s when researchers were searching for an alternative synthetic material for natural textiles. Dacron is a branded synthetic polyester produced by DuPont. Furthermore, by the late 1950s, researchers managed to prepare stretched thin sheets of PET by extrusion molding to create films that are now extensively used as X-ray or photographic films. The technology of PET production and molding achieved much progress by the 1970s towards the formation of strong and lightweight bottles. PET is highly resistant to attack by microorganisms or reaction with food products, and this privilege places it on top of packaging materials used in the processing of foods, beverages, and pharmaceuticals. Based on these facts, health-safety agencies around the world have approved PET as safe for use with foods and beverages for many decades. The massive production of polymeric packaging materials around the world has implemented research work for their recycling to manage their increasing waste volume. PET is at the top of all recyclable polymer wastes due to its easy recycling and high sustainability, and it is the most recycled plastic in the United States and worldwide. PET is recycled into shorter chain oligomers and co-reacted with different raw reactant materials with various functional groups to produce new polymeric materials or reproduce PET back and used in forming containers for foods, beverages, pharmaceutical products, fibres for carpets and clothing, automotive parts and construction materials. Finding solutions to reduce the massive volume of discarded plastic products became mandatory for managing the growing portion of municipal solid waste (MSW). ${ }^{1,2}$ Statistical data revealed that the global production of PET increased from 41.56 million metric tons in 2014 to 73.39 million metric tons in 2020. Another instance of the high 
consumption of PET in the production of beverage bottles was recorded in 1987, where more than 700 million pounds were in this industry alone. ${ }^{3}$ The effective utilization of PET waste is considered of high economic and technological importance. PET can be depolymerized by different chemical methods, which is attributed to the relative chemical reactivity of its ester functional group, where its chemical reactivity is ranked among other carboxylic acid derivatives as follows: acid halides > acid anhydrides > esters > amides. ${ }^{4}$ The covalent bonding of the $\mathrm{C}$ atom to the more electronegative $\mathrm{O}$ atom from both ends in the ester linkage $(\mathrm{O}=\mathrm{C}-\mathrm{O}-)$ creates a permanent dipole with a positive charge $(+\delta)$ on the $\mathrm{C}$ atom and a negative charge $(-\delta)$ on the acyl 0 atom, and this polarity of the ester carbonyl group makes it highly reactive towards nucleophilic addition reactions. Nucleophilic reagents could be neutral or negatively charged. Examples of neutral nucleophiles are Lewis bases in their nature, such as amines, alcohols, glycols, etc. $\left(\mathrm{R}-\mathrm{NH}_{2}, \mathrm{R}-\mathrm{OH}, \mathrm{HO}-\mathrm{R}-\mathrm{OH}\right)$, while negatively charged nucleophiles are anions such as cyanides, chlorides, hydroxides, etc. $\left(-\mathrm{CN}^{-}, \mathrm{Cl}^{-},-\mathrm{OH}^{-}\right)$. This fact opens the potential for many innovative depolymerization processes for converting PET waste into numerous value-added degradation products. PET waste can be depolymerized into shorter chain oligoesters by alcoholytic degradation as a Lewis base nucleophile. ${ }^{5-11}$ PET can be depolymerized by other Lewis base nucleophiles, such as water (hydrolysis), amines (aminolysis), and glycols (glycolysis). Methanolysis has been evaluated as a method for recycling photographic and X-ray films, with more than $158 \times 10^{6} \mathrm{~kg}$ converted since $1977 .{ }^{12}$ However, large-scale degradation by methanolysis was not financially efficient. ${ }^{13}$ For the purpose of highly efficient methanolysis, high-pressure reactors should be involved in the process. ${ }^{14,15}$ Dupont et al. ${ }^{16,17}$ reported on the degradation transesterification of PET scraps by 2-ethyl-1-hexanol for the purpose of synthesizing dioctyl terephthalate (DOTP) plasticizers for flexible PVC. PET waste can be used in molded or extruded articles after repalletizing. ${ }^{18}$ However, repalletizing might degrade the polymer, adversely affecting its properties. Blending PET waste with raw polymer adversely affects the color and melt viscosity of the final product. ${ }^{19}$

Depolymerization of PET by glycolysis into oligoester polyols that can be further converted into new value-added products is the applied method in this work. The glycolysis process was carried out in the presence of manganese acetate as a transesterification catalyst in the temperature range of 180-200 ${ }^{\circ} \mathrm{C}^{20-23}$. The produced polyols are used as reactive starting materials for other industries, such as polyurethanes $\mathrm{PU}^{24}$, unsaturated polyesters ${ }^{25,26}$ and modified unsaturated polyesters. ${ }^{27-30}$

\section{Experimental}

\section{Materials}

Diethylene glycol DEG, manganese acetate catalyst Mn-ac, toluene diisocyanate TDI, acryloyl chloride ACl, 2-benzyl-2-dimethylamino-1-(4-morphlinophenyl)-1-butanone BDMB, diethylene glycol diacrylate DEGDA, diethylene glycol dimethacrylate DEGDMA, triethylene glycol dimethacrylate TEGDMA and styrene Sty were all purchased from Aldrich Chemical Co. and used without further purification. 


\section{Results And Discussion}

This work is a continuation of our previously published series of work ${ }^{27-30}$ on the modification of oligoester polyols derived from the glycolysis reaction of PET waste. In our previous work, PET waste was depolymerized at different molar ratios of PET:DEG, and the obtained oligoester polyols were modified to acrylated and methacrylated UV curable oligomers ${ }^{29,30}$. In this work, the obtained oligoester polyols were reacted first with half equivalents of TDI to insert a urethane moiety into the backbone of the polymer chain followed by modification of the terminal hydroxyl groups to acrylates. The purpose of incorporating the urethane nucleus into the polymer structure was to improve the mechanical characteristics of the final cured films. Figure 1 shows all the steps of glycolysis of PET and chemical reaction with TDI and modification with $\mathrm{ACl}$ to obtain the modified acrylated urethane-oligoester GLYUA.

\section{Glycolysis of PET Waste}

PET was first depolymerized by diethylene glycol DEG at a molar ratio of PET:DEG of 1:2.15 based on the molecular weight of the terephthalate ethylene glycol repeating unit $\left(\mathrm{C}_{10} \mathrm{H}_{8} \mathrm{O}_{4}\right)$ of $192.17(\mathrm{~g} / \mathrm{mol})$. Depolymerization of PET was carried out in a continuously stirred three-necked flask equipped with a condenser, thermometer, and nitrogen inlet to avoid any undesirable oxidation side reactions in the presence of atmospheric oxygen at elevated temperatures. The temperature of the reaction was kept constant in the range of $180-200^{\circ} \mathrm{C}$ for a period of 4 hours $^{31,32}$. After completion of the glycolysis reaction, the product was cooled to room temperature and dissolved in an appropriate amount of dichloromethane DCM to lower the viscosity of the glycolyzed product GLY and further purified by liquidliquid chromatography LLC through phase separation between solvent-nonsolvent phases using distilled water as the polar nonsolvent to remove any unreacted glycol and catalyst ${ }^{27-30}$ DCM organic layer containing solubilized oligoester polyol GLY. The product was then collected and dried from DCM by a rotary evaporator and tested for acid number and hydroxyl value by End Group Analysis EGA. The eventually collected GLY was reacted with half equivalent of TDI based on the determined hydroxyl value to form a hydroxyl terminated urethane-oligoester GLYU. The remaining half equivalence of terminal hydroxyl groups of GLYU were modified into acrylate groups through acrylation reaction ${ }^{33,34}$ to prepare the modified product of acrylated urethane-oligoester GLYUA, which was copolymerized with different comonomers and tested for their mechanical properties. GLYUA was copolymerized with different UV curable comonomers, such as diethylene glycol diacrylate DEGDA, diethylene glycol dimethacrylate DEGDMA, triethylene glycol dimethacrylate TEGDMA and styrene Sty. Copolymerization and crosslinking reactions were carried out under UV irradiation using 2-benzyl-2-dimethylamino-1-(4-morphlinophenyl)-1butanone BDMB as a photoinitiator, and cured products were tested for their mechanical properties.

\section{Molecular Weight Determination by End Group Analysis EGA}


The number average molecular weights $<M_{\mathrm{n}}>$ of the obtained GLY were calculated according to the end group analysis method by determination of the acid number $(A)$ and hydroxyl value $(B)(\mathrm{mg} \mathrm{KOH} / \mathrm{g}$ sample) ${ }^{35-38}$ and substituting into Eq. (1) ${ }^{39,40}$, where $n$ refers to the average number of hydroxyl groups/oligomer molecule.

\section{$M_{\mathrm{n}}=(n \cdot 56.1 \cdot 1000) /(A+B)$ \\ 3. Molecular Weight Determination by Gel Permeation Chromatography (GPC)}

GPC analyses were performed on SEC Inst., using THF as a solvent at ambient temperature. For measurement, a $20 \mu \mathrm{L}$ sample was injected at a flow rate of $1 \mathrm{~mL} / \mathrm{min}$. using waters $5 / 5 \mathrm{HPLC}$ pump. Two successive columns (PSS SDV linear XL $5 \mu, 8 \times 600 \mathrm{~mm}$ ) and (PSS SDV $100{ }^{\circ} \mathrm{A} 5 \mu, 8 \times 600 \mathrm{~mm}$ ) were applied. Detection was performed on a Waters 410 Differential Refractometer using a linear UVIS-205 absorbance detector. We have investigated the structure of the obtained GLY under the same chemical conditions in our previous work ${ }^{27-30}$, and it was shown that GLY contains $(n=3)$ hydroxyl groups per oligomer molecule, and the measured $\left\langle M_{\mathrm{n}}>\right.$ from EGA and GPC lies between 480-500 ( $\left./ \mathrm{mole}\right)$.

\section{Synthesis of Urethane-Oligoesters}

Based on the measured hydroxyl value ( $B$ ) by EGA, GLY was reacted with an equivalent of TDI to form urethane-oligoester GLYU with half equivalent of its terminal hydroxyl groups available for modification into acrylate terminal groups. Reaction with TDI was carried out by heating at $70^{\circ} \mathrm{C}$ in a vacuum oven for 12 hours to ensure complete reaction of cyanate groups with equivalent hydroxyl groups.

\section{Synthesis of the Urethane-Ester Acrylated Oligomer}

The terminal hydroxyl groups of the urethane-oligoester GLYU were modified to acrylate groups by reaction with acryloyl chloride according to a published procedure ${ }^{33,34}$. A $250 \mathrm{~mL}$ round-bottom threenecked flask equipped with a magnetic stirrer and a nitrogen sparge tube was charged with $150 \mathrm{~mL}$ tetrahydrofuran (THF), $x$ mol of purified GLYU and $3 x$ mol triethylamine. After cooling the mixture under a nitrogen spurge to $0{ }^{\circ} \mathrm{C}$ with an ice bath, the calculated equivalent moles of acryloyl chloride were added dropwise via an additional funnel over a $30 \mathrm{~min}$ time span. The mixture was kept at $0{ }^{\circ} \mathrm{C}$ for $3 \mathrm{~h}$ and allowed to reach room temperature and remain for an additional $3 \mathrm{~h}$. After filtration and removal of the THF under vacuum, the crude product was dissolved in DCM. The obtained DCM solution was washed out with $1.0 \mathrm{M}$ aqueous $\mathrm{NaOH}$ and saturated aqueous $\mathrm{NaCl}$, three times in each case, followed by drying the organic layer over anhydrous $\mathrm{Na}_{2} \mathrm{SO}_{4}$. The final solution was concentrated and purified by passage over an anhydrous silica column using a $\mathrm{CH} 3 \mathrm{OH} / \mathrm{CH} 2 \mathrm{Cl} 2(50: 50 \mathrm{v} / \mathrm{v})$ mixture as a mobile phase. ${ }^{41,42}$ The urethane-ester acrylated oligomer GLYUA was then copolymerized with UV curing with other comonomers and tested for Young's modulus, \% elongation and break strength.

\section{UV Curing}


The finally synthesized and purified acrylated urethane-oligoester GLYUA was then cured solely under UV irradiation and as a comonomer with other UV curable monomers or a mixture of monomers. Curing was carried out by the addition of $2 \mathrm{wt} .-\%$ BDMB and UV irradiation at $\lambda=254 \mathrm{~nm}$ at an output intensity of 400 $\mathrm{W} /$ inch. The speed of the conveyor belt was $5 \mathrm{ft} / \mathrm{min}$ for a traveled distance of $I=33.0$ inches. Cured films were obtained by casting liquid mixtures of monomers onto glass plates for a definite thickness controlled by using a steel thickness guard.

\section{Essence Of The Measured Mechanical Properties}

The mechanical characteristics of the tested cured films were recorded in terms of the stress/strain relationship. Young's modulus measures the resistance of a material to elastic (recoverable) deformation under loading. A stiff material exhibits a high Young's modulus and changes its shape only slightly under elastic loads. A flexible material, on the other hand, shows a low Young's modulus and changes its shape considerably, similar to rubbery materials. A stiff material requires high loads to elastically deform, which requires high loads to permanently deform (or break) it. The stiffness of a material means how much it deflects under a given load. Young's modulus is equal to elastic stress/strain ${ }^{43-45}$. Young's modulus has the same units of stress: $\mathrm{N} / \mathrm{m}^{2}$, or Pascals $\left(1 \mathrm{~Pa}=1 \mathrm{~N} / \mathrm{m} 2 ; 1 \mathrm{GPa}=1000 \mathrm{~N} / \mathrm{mm}^{2}\right)$. Experimental data for Young's modulus and break strength of the cured samples are expressed in units of GPa. As presented on Table 1.

The measured values of Young's modulus for the UV-cured samples were between 1.5 and $2.6 \mathrm{GPa}$ ), which represents a good achievement when comparing samples PU1-PU4 with similar samples from our previous work that did not contain urethane moieties, which showed Young's modulus values of (0.9-1.6 $\mathrm{GPa})^{29,30}$. PU1 is the control sample for comparing the effect of the comonomer ratio on the measured mechanical properties, and it was cured solely under UV irradiation. The values of Young's modulus and break strength for the control sample PU1 were the lowest, but its \% elongation was the highest when compared with other samples, which indicates its highest flexibility. Samples PU2-PU4 were copolymerized with other comonomers at a ratio of $50 \%$ by weight, which resulted in pronouncedly improved values of Young's modulus and break strength, but the \% elongation dropped to lower values, indicating the imparted stiffness to the samples. The sum of $50 \%$ by weight ratio of the comonomer was varied to include $25 \%$ by weight of styrene monomer to incorporate the polystyrene segments into the final crosslinked structure of samples PU2-2, PU3-2 and PU4-2 and this did not change much of the values of Young's modulus but led to improvement in the break strength (MPa). The prevailing data in Table 1 indicated the success of our endeavor of incorporating a urethane moiety into the chemical structure of the glycolyzed product of PET waste. Second, copolymerization with other UV curable monomers helped drop the viscosity of the comonomer mixture, which made it more applicable in making cured films and further improved the mechanical characteristics of Young's modulus (GPa) and break strength (MPa) to much higher values than the control sample. Styrene was incorporated as a comonomer because its segments are glassy and rigid, which should improve the mechanical strength and was established as indicated by the measured value of break strength. 


\section{Conclusion}

Chemical degradation of PET waste by glycolysis reaction into polyol oligoesters is an interesting endeavor for creating new and innovative value-added products. Steps of modification of these polyol oligoesters were achieved by inserting urethane blocks into the chemical structure of the glycolyzed products in addition to modifying the terminal hydroxyl groups into UV curable acrylate moieties GLYUA. The mechanical properties of GLYUA cured solely with UV light were noticeably improved by copolymerization with 50\% DEGDA, DEGDMA and TEGDA. The mechanical properties were further improved to the highest values by incorporation of $25 \%$ styrene monomer by weight.

\section{Declarations}

\section{Acknowledgement}

This research work was carried out at the University of Alabama, chemistry department under a project funded by the U. S. Environmental Protection Agency under grants R826728 and R827121. The contents do not necessarily reflect the views and policies of the U.S. EPA, nor does the mention of trade names or commercial products constitute endorsement or recommendation for use. The author cordially expresses his gratitude and acknowledgement for the continuous research support and help offered by Professor Dave E. Nikles and the staff at the center for materials for information technology at the University of Alabama.

\section{Data availability}

The data sets generated and analyzed during the current study are available at the following journals online repository; Macromolecular Materials and Engineering MME and Polymer International PI.

https://doi.org/10.1002/1439-2054(20011101)286:11<695::AID-MAME695>3.0.C0;2-V

https://doi.org/10.1002/1439-2054(20020501)287:5<353::AID-MAME353>3.0.CO;2-H

https://doi.org/10.1002/pi.818

\section{References}

1. B. G. Lipták, "Municipal Solid Waste Disposal in the 1990s", Chilton Book Company, 1991, p. 318.

2. C. R. Rhyner, L. J. Schwartz, R. B. Wenger and M. G. Kohrell, "Waste Management and Resource Recovery", CRC Press, Inc., 1995, p. 109.

3. A. K. Bledzki, ed., "Recycling of Polymer Material", WNT, Warsaw (1997).

4. J. Scheirs and T. E. Long, "Modern Polyesters: Chemistry and Technology of Polyesters and Copolyesters" $1{ }^{\text {st }}$ ed., Wiley 2003

5. J. Mleziva, "Polyesters", Technika, Sofia 1969. 
6. B. V. Petuhov, "Polyester Fibers”, Khimia, Moscow 1976, pp. 81-181.

7. K. Todorov, K. Kolev, I. Iranov, A. Todorova and A. Georgieva, Bulgarian Authorship Certificate No. 47724 (1989).

8. C. W. Neale, N. C. Hilyard and P. Barber, Conservation and Recycling, 1983, 6, 91.

9. J. W. Jensen, J. L. Holman, and J. B. Stephenson, "Recycling and Disposal of Waste Plastics", Ann Arbor Science 1974, Chap. 7.

10. J. Milgrom, "Polyethylene terephthalate. In Plastics Recycling", Ehrig, RJ (ed.), Hanser Publisher; Munich (1989).

11. L. M. Warren and R. Burns, Plastics Technology, 1988, 6, 41

12. H. Van, ed., "Kodak begins recycling film", Chem. Mark. Rep., 1990, No. 9 (Mar. 5), 237.

13. R. Martino, ed., Modern Plastics, Aug. 1990, p. 126.

14. U.S. Pat. 3, 037, 048 (1962), inv.: R. Lotz.

15. U.S. Pat. 4, 578, 502 (1962), inv.: W. J. G. Cudmore.

16. L. A. Dupont and V. P. Gupta, J. Vinyl Technol., 1993, 15, 100.

17. U.S. Pat. 5, 101, 064 (1992), invs.: L. A. Dupont and V. P. Gupta.

18. U. S. Pat. 4, 164, 880 (1979), invs.: W. H. Cobbs, M. J. Stewart.

19. M. Sitting, in: "Organic and Polymer Waste Reclaiming Encyclopedia", Noyes Data Corpn., New Jersey 1981, p. 41.

20. J.-W. Chen and L.-W. Chen, J. Appl. Polym. Sci., 1999, 73, 35.

21. F. Hubert, G. Durand and G. Tersac, J. Appl. Polym. Sci., 1999, 72, 329.

22. N. Halacheva and P. Novakov, Polymer, 1995, 36, 867.

23. S. Baliga and W. T. Wong, J. Polym. Sci., 1989, A27, 2071.

24. U. R. Vaidya and V. M. Nadkarni, J. Appl. Polym. Sci., 1988, 35, 775.

25. U. R. Vaidya and V. M. Nadkarni, Ind. Eng. Chem. Res., 1987, 26, 194.

26. U. R. Vaidya and V. M. Nadkarni, Ind. Eng. Chem. Res., 1988, 27, 2056.

27. M. S. Farahat, A. A. Abdel-Azim and M. E. Abdel-Raowf, Macromol. Mater. Eng., 2000, 283, 1.

28. M. S. Farahat, Polym. Int., 2002, 51, 183.

29. M. S. Farahat and D. E. Nikles, Macromol. Mater. Eng., 2001, 286, 695

30. M. S. Farahat and D. E. Nikles, Macromol. Mater. Eng., 2002, 287, 353

31. S. N. Tong, D. S. Chen and L. Z. Chung, Polymer, 1983, 24, 469.

32. K. S. Rebeiz, D. W. Fowler and D. R. Paul, J. Appl. Polym. Sci., 1992, 44, 1649.

33. F. Gao, B. M. Culbertson and S. R. Schricker, Polym. Prep., 2000, 4, 125.

34. K.-D. Ahn, C.-M. Chung and Y.-H. Kim, J. Appl. Polym. Sci., 1999, 71, 2033.

35. S. Siggia, "Quantitative organic analysis via functional group analysis", $3^{\text {rd }}$ ed., John Wiley and Sons, New York 1963, p. 8. 
36. R. S. Stetzler and C. F. Smullin, Anal. Chem., 1962, 34, 194.

37. J. S. Fritz and G. H. Schenk, Anal. Chem., 1959, 31, 1808.

38. D. F. Fritz, A. Sahil, H-P. Keller and E. Cz. Kovats, Anal. Chem., 1979, 51, 7.

39. W. R. Sorenson, W. Sweeny and T. W. Campbell, "Preparative Methods of Polymer Chemistry", $3^{\text {rd }}$ ed., Wiley-Interscience, New York 2001, p. 358.

40. G. Odian, "Principles of Polymerization", $2^{\text {nd }}$ ed., John Wiley and Sons, New York 1981, p. 40.

41. A. Priola, G.F. Ferrero and G. Malucelli, Polymer, 1993, 34, 3653.

42. G. Malucelli, G. Gozzelino, R. Bongiovanni and A. Priola, Polymer, 37, 2565.

43. ASTM D638 - Standard Test Method for Tensile Properties of Plastics

44. ASTM D882 - Standard Test Method for Tensile Properties of Thin Plastic Sheeting

45. ASTM E111 - Standard Test Method for Young's Modulus, Tangent Modulus, and Chord Modulus

\section{Tables}

Due to technical limitations, table 1 is only available as a download in the Supplemental Files section.

\section{Figures}




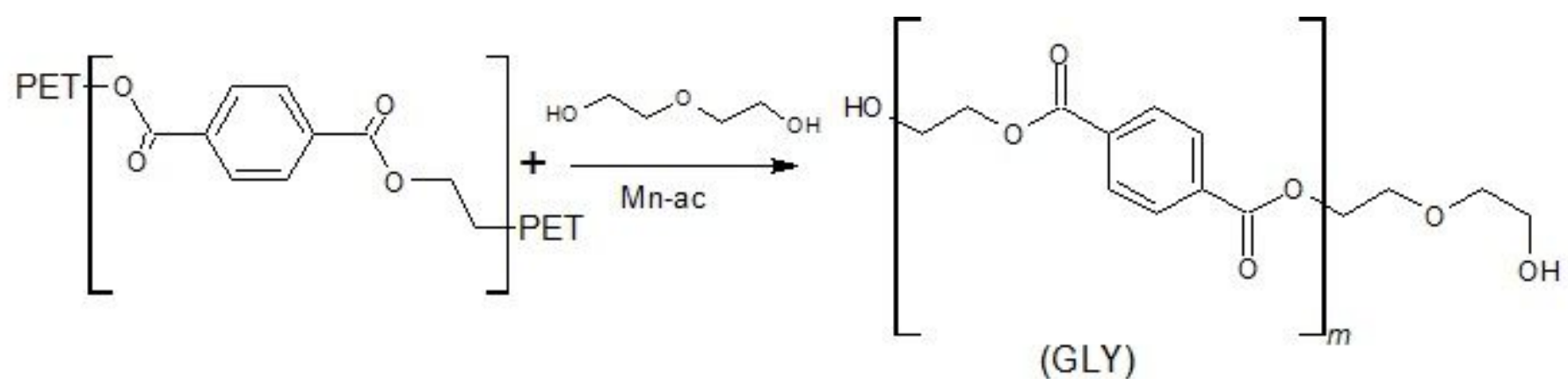

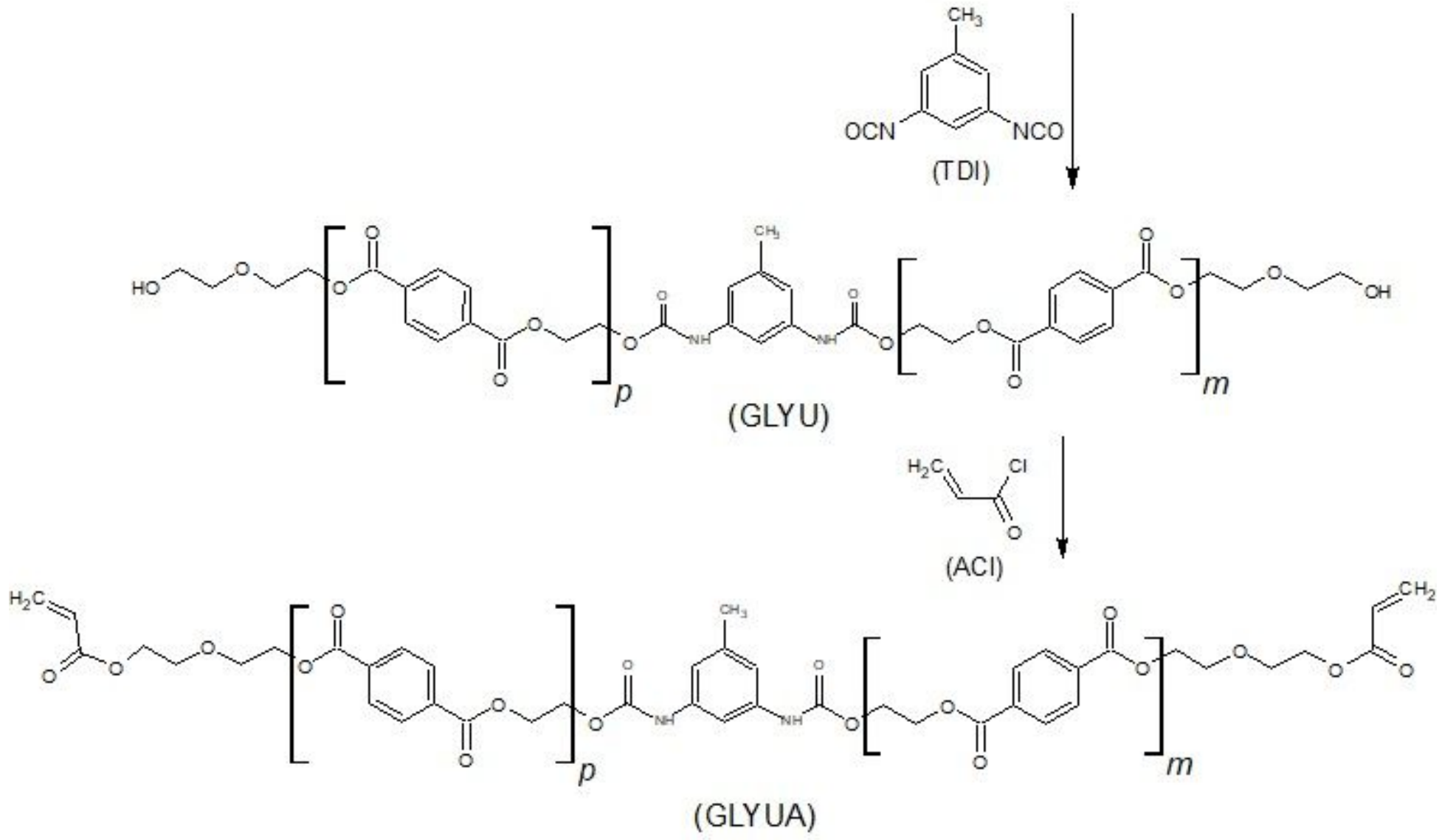

Figure 1

Sketch of the predicted chemical structures of GLY, GLYU and the modified GLYUA monomer

\section{Supplementary Files}

This is a list of supplementary files associated with this preprint. Click to download.

- Table1.jpg 